Perspective

\title{
Psychological Considerations in the Etiology and Pathophysiology of Migraines
}

\author{
Niamh Flynn *
}

Suite 29 The Galway Clinic, Doughiska, Galway; E-Mail: Niamh.flynn@galwayclinic.com

* Correspondence: Niamh Flynn; E-Mail: Niamh.flynn@galwayclinic.com

Academic Editor: Bart Ellenbroek

Special Issue: The Pathophysiology and Treatment for Migraine

OBM Neurobiology

2021, volume 5 , issue 2

doi:10.21926/obm.neurobiol.2102092
Received: March 18,2021

Accepted: April 21,2021

Published: May 02,2021

\begin{abstract}
Although studies to understand the pathogenesis of migraines are in progress, no theory has adequately explained the etiology and pathophysiology of migraines to date. This has affected the development of treatment strategies for migraineurs. Currently, the pharmacological treatment for migraines provides both acute and prophylactic options to patients based on a biomedical model of pain. However, patients' adherence to oral migraine preventive medication (OMPM) is poor, and their persistence is even lower when they cycle through a variety of OMPMs [1]. Although our understanding of the pathophysiology of migraines within the context of the current biopsychosocial model of pain has advanced in recent years, there is a need to better understand the role of social and psychological factors in the pathophysiology of this debilitating disease, which would pave the way for the development and acceptance of more diverse and inclusive treatments. In this review, we provide an overview of the various theories that purport to explain the pathogenesis of the headache phase of migraines, examine the usefulness and shortcomings of these theories, and investigate how psychological considerations may help develop treatments to assist migraine sufferers in managing their headaches better.
\end{abstract}

\section{Keywords}

Biopsychosocial model of pain; migraine; pathophysiology; anatomy; psychology of pain; vascular theory; neurotransmitter theory; brainstem theory; neurovascular theory

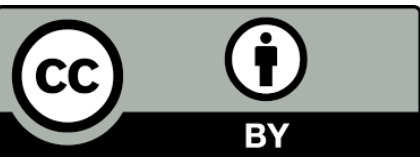

(C) 2021 by the author. This is an open access article distributed under the conditions of the Creative Commons by Attribution License, which permits unrestricted use, distribution, and reproduction in any medium or format, provided the original work is correctly cited. 
Several potential theories have explained the pathogenesis of migraines [2], including vascular theory [3, 4], neurovascular theory [5], neurotransmitter theory, and brain stem theory. Although various explanations exist for the pathogenesis of migraines, no definitive explanation has yet been established [6, 7], and none of the theories account for all symptoms that occur in a single attack [8]. Edmeads [9] suggested that researchers require stepping outside the polemic as to whether migraines are primarily a vascular or neurological dysfunction and consider the role of neurotransmitters as being a link between neural and vascular systems and the impact neurotransmitters have on neural and vascular systems as well as the gastrointestinal tract.

While considering migraines within a framework of the current biopsychosocial model of pain, the impacts of thoughts, beliefs, and the environment on the pathogenesis of this disease should also be considered.

\section{Vascular Theory of Migraines}

Historically, migraines were believed to be primarily vascular in nature [4]. This vascular theory persisted up to the mid-to the late 20th century. This theory was based on three factors: (1) pain is registered in large cranial vessels, proximal cerebral vessels, large veins, venous sinuses, and dural arteries; (2) during a migraine attack, the carotid artery becomes engorged; and (3) ergotamine, a vasoconstrictor, was the most effective antimigraine drug [10]. Since the late 20th century, other explanations for the pathogenesis of migraines have emerged.

\section{Neurovascular Theory of Migraines}

Goadsby [11] reported that based on the observation by Wolff that the migraine aura was the result of vasoconstriction and the ensuing headache was a result of reactive vasodilation, migraines during the 1960s and 1970s were considered a vascular phenomenon. The experiments by Graham and Wolff, instigating the vascular theory (as cited in Hines [12]), involved administering ergotamine (a vasoconstrictor) to migraineurs. When this alleviated migraines, they administered histamine (a vasodilator) and subsequently reproduced the headache.

Goadsby stated that this vascular theory was later rejected for multiple reasons. Not everyone who had migraines had an aura. Furthermore, nonvascular acute medications were effective in tackling migraines. He discussed the brain stem hypothesis for migraines, which developed as a result of an observation that brain stem aminergic neurons, for example, noradrenergic neurons of the locus coeruleus, could cause the unusual features typical of the aura phase in migraines, such as sensitivity to light and sound and unilateral head pain. Goadsby outlined that this notion was supported by positron emission tomography (PET) studies, which demonstrated that activations in the rostral brain stem lingered after a migraine attack but were not present in the period between migraines, that is, interictally [13]. Weiller et al. [13] reported that these findings supported the concept that the pathogenesis of migraines was related to an imbalance in the activity between brain stem nuclei regulating antinociception and vascular control. Goadsby concluded that clinicians could confidently inform patients that the disorder was localized in the brain and it is a brain function disorder and not a disorder of blood vessels, as was previously speculated. Blau [5] was 
less inclined to discount the vascular element of migraines and instead defined migraine as a "primary neurological disturbance with secondary vasomotor changes" (p. 437). He considered migraine theories to be incomplete and used the analogy of a house where theories are based on the roof, and both the main building (the symptoms of migraines, e.g., irritability, mood changes, and tiredness) and the foundations (prodromes and auras, for example) are neglected.

Blau [5] listed eight points that supported the neurological pathogenesis of migraines and proposed that the vasomotor features were secondary to neural stimulation. Blau explained that this was consistent with two theories first identifiedsupported by Liveing [14]. The first of these was based on changes in blood vessels, evident from the color of the patient's skin. This was attributed to flushing of the skin due to dilation of blood vessels and pallor of the skin due to contraction of the arteries. As per the second theory, migraine was described as a primary disorder of the nerve cells of the brain leading to occasional peculiar vasomotor disturbances. The eight points described by Blau [5] to support the latter theory are as follows:

- Migraine attacks can be precipitated internally by stimuli from the nervous system (stress or sleep disturbance) or externally from the environment (light), exciting the neural pathways.

- The hypothalamus is involved in neurological prodromes such as tiredness or food cravings.

- The classic sensory aura cannot be explained by vascular functioning.

- Neurological symptoms such as lack of focus and tiredness continue after the prodrome and into the headache phase of migraines.

- Neurological symptoms persist after the headache phase of migraines.

- Sleep is a solution for many migraine attacks.

- Changes in cerebral blood flow during a migraine with an aura cannot be explained in vascular terms.

- Electroencephalogram (EEG) changes have been found before, during, and after the headache phase of migraines.

Blau [5] concluded that both vascular and neurological hypotheses should be considered, and the implication of altered neural transmission in migraines warranted further investigation.

\section{Neurotransmitter Theory of Migraines}

Serotonin-based medication is currently prescribed to migraineurs, with evidence indicating that this neurotransmitter is most commonly implicated in migraine pathophysiology [15]. Interest in serotonin-based treatment stems from observations that drugs that reduce the levels of serotonin increase the number of migraine attacks, and intravenous serotonin has been shown to abort migraines [2]. Low serotonergic disposition has been witnessed among migraineurs, leading researchers to propose that migraines are a consequence of a neurochemical imbalance [15]. Yaknitsa et al. [16] observed that serotonin was moderated by neuronal calcium channels and advised that dysfunction in calcium channels could compromise the release of serotonin and subsequently predispose individuals to migraine.

D'Andrea et al. [17], in their review, examined the biochemical studies. They found evidence for metabolic abnormalities during the synthesis of neurotransmitters and neuromodulators. They attributed the abnormalities to impaired mitochondrial function and high levels of glutamate in the central nervous system (CNS) of migraineurs. This led them to hypothesize that migraine attacks were triggered by a process that started in the frontal lobe and progressed to abnormal activation 
of nuclei in the pain matrix. The idea that there is a "pain matrix," that is, pain emerging from multiple areas of the brain rather than being the existence of one specific locus of pain, is widely accepted in the pain literature [7]. Based on neuroimaging studies that reported altered functional connectivity between brain stem pain-modulating circuits and limbic centers in the brains of migraineurs, Maizels et al. [7] also proposed the existence of a "limbic" influence in the etiology of migraines. They suggested a bidirectional interaction between pain and mood and reported that neurolimbic dysfunction can increase when migraines become chronic.

\section{Brain Stem Theory of Migraines}

Benoit [2] reported that abnormal brain stem activity may be a part of the pathophysiology underlying migraines. Welch et al. [18] proposed that the periaqueductal gray (PAG) region of the brain stem may be one possible "generator" of migraine attacks. This hypothesis was supported by previous observations made by Raskin et al. [19]. Raskin et al. [19] reported that 15 patients with no history of headaches who underwent the placement of an electrode in the PAG region subsequently reported headaches with migraine-type features a few days postimplantation. They concluded that disturbance in this region of the brain could initiate migraines.

A more recent study by Mathew [20] compared the brains of chronic migraineurs (CMs) with those of episodic migraineurs (EMs). He found more advanced changes in specific structural areas of the brain. One of these changes was the accumulation of iron in the PAG matter of CMs. Mathew [20] observed that this accumulation was different from that in EMs, and these changes correlated with the duration of the migraine disorder. Other physiological differences between the brains of EMs and CMs included greater cortical excitability in CMs and a higher incidence of cutaneous allodynia. Mathew [20] explained that cutaneous allodynia, which correlated with migraine frequency and duration of illness, was a marker of central pain sensitization, which generated free radicals and damaged the PAG. Reisman and Fuller [21] noted that repeated attacks of headaches or auras caused iron accumulation in the PAG, which, in turn, impaired the antinociceptive system that controls the trigeminovascular system (TGVS), the part of the brain associated with migraine pain [22]. Further support for the brain stem hypothesis comes from the findings that regional cerebral blood flow (rCBF) increases in several areas of the dorsal rostral brain stem during a migraine attack, indicating defective activity [22]. The researchers suggested that this defective activity would either "trigger a migraine (brainstem generator of migraine) or contribute to central hyperexcitability of trigeminal pathways" (p. 387). Pietrobon and Striessnigh [22] explained that this was important because two of the issues, which remained misunderstood about migraines, were the primary cause of migraines activating the TGVS, and the process of pain generation after the TGVS was activated.

\section{Other Theories of Migraine Etiology}

Studies investigating the role of the patent foramen ovale (PFO) and the role of genetics in migraines could be relevant in the future understanding of migraine pathophysiology. Harder [23] described the unusual findings of a Swiss neurologist who treated a stroke patient by shutting the PFO, a residual tunnel in the heart. The patient, a lady in her 30s, wrote to the surgeon to thank him for a subsequent decrease in her migraines. In his article, Harder [23] described how the PFO could act as a valve and added that blood clots occasionally passed through the PFO to the brain and 
instigated a stroke. He cited further examples of cases where cardiologist Peter Wilmshurst found several scuba divers whose PFOs were surgically closed to prevent decompression sickness; they reported having fewer migraines after the operation. As the research in this area was related to individuals who had other problems, the author suggested further investigation before the procedure could be considered a treatment option for migraines. Reisman and Fuller [21] commented on the only randomized controlled trial (RCT) examining PFO closure in migraine patients and compared the results with those of retrospective single-center studies. The RCT did not confirm the results from the retrospective studies, and Reisman and Fuller [21] attributed this to slow enrolment in the RCT and the narrow inclusion criteria, which excluded many migraineurs. Nonetheless, the researchers cautioned that the impressive results from single-center nonrandomized trials should not be ignored and that PFO closure had the potential to reduce the functional disability of migraines.

Although some research has been conducted in the area of genetics and migraines, migraine genes have been, and continue to be, difficult to identify [24] and consequently treat. In a review of the literature, D'Andrea et al. [17] noted that although specific mutations of genes involved in the pathogenesis of migraines were yet to be determined, the polymorphisms and mutations that regulated neurotransmitter metabolisms and ion channels in the CNS were the main biological factors influencing migraines.

Several migraine medications are available that have cardiovascular side effects. The drive to develop safer and more effective migraine treatments was instrumental in the development of calcitonin gene-related peptide (CGRP) antagonists [25].

Cortical spreading depression (CSD) is an electrophysiological phenomenon characterized by a wave of excitation followed by inhibition in cortical neurons [26]. It is believed to be central to the pathophysiology of the migraine aura and is a complex affair, involving changes in neural and vascular functions [27]. CSD can activate the trigeminal nociceptive system both peripherally and centrally [28]. Together, these observations have provided an opportunity to better understand how new treatments for migraines can be developed, for example, drugs that target the CGRP, a primary neuropeptide of the trigeminal system, and a vasodilatory peptide [28]. To date, the number of side effects of anti-CGRP therapy for migraine attacks are limited and predominantly confined to gastrointestinal-related disorders [29]. Although monoclonal antibodies targeting the CGRP pathway have shown some efficacy in preventing migraines with good tolerance [30], the long-term effects of CGRP monoclonal antibodies are as yet unknown.

\section{Psychology and Headaches}

Clinicians are recommended to consider the various factors that affect the development of a headache, the course and severity, and the subsequent disability to minimize the frequency of attacks, and reduce the severity and limit the effect of headaches on functioning [31]. Nicholsan et al. [31] identified two types of cognition as being specifically influential concerning headaches, namely, locus of control and self-efficacy. The researchers also proposed that the negative effect in headache patients results from a combination of anxiety, depression, and anger. The co-occurrence of depression and anxiety in migraineurs has frequently been reported, for example, Merikangas et al. [32] and Breslau et al. [33]. Notably, Brown et al. [34] suggested a complex bidirectional association between mood disorders and migraines, as experiencing a migraine can, in and of itself, 
cause psychological stress. They stated that pain management strategies could assist people in managing their pain more effectively and recommended that a combination of medical and psychological approaches should be employed to treat migraines rather than adopting a single approach.

Hamelsky and Lipton [35] suggested that the comorbid relationship between migraine, depression, and anxiety disorders could be clinically significant, and treatment of one condition could prevent the progression to one or both of the other two conditions. For the treatment of patients who suffer from both depression and migraines, the authors suggested that treatment options that addressed both of these conditions should be considered. A prospective cohort study with a follow-up of 8 years involving 9,288 participants conducted by Swanson et al. [36] explained the association between migraines and depression by stress. Swanson et al. [36] initially reported several types of stressors, including childhood trauma, chronic stress, work stress, change in social support, financial strain, recent marital problems, and recent unemployment. They were interested in finding out, which particular stressors might confound the migraine-depression association. Their results showed that chronic stress was strongly predictive of the onset of migraines and depression, whereas recent changes in the marital relationship status and recent employment changes were not strongly predictive of either depression or migraines. They pointed out that these results could develop intervention strategies and recommended that effective stress-management strategies for migraineurs and individuals suffering from depression could significantly prevent and may positively impact the economic burden that these disorders have on society. In addition, Hernyk-Gutt and Rees [37] and Kim et al. [38] found that emotional stress could act as a precipitating factor in migraines. To test the hypothesis that psychological factors contributed to the etiology of migraines, Henryk-Gutt and Rees [37] matched a random sample of 50 male and 50 female migraineurs with similar groups who suffered from nonmigraine headaches and groups that did not experience headaches. Their findings indicated that more than $50 \%$ of the attacks documented during a 2month observation were related in time to a stressful event and $50 \%$ of the random sample experienced migraines for the first time during a period of emotional stress. Henryk-Gutt and Rees [37] also suggested an increase in the activity of the autonomic nervous system in migraineurs and noted the importance of this factor in the development of migraine attacks.

Brown et al. [34] reported that although a migraine "is a biological and not a psychological entity" (p. 78), it was important to acknowledge the role of psychological factors in treating migraines. Brown et al. [34] listed psychological factors that could influence migraines in four ways: (1) migraines can be triggered by psychological stressors, (2) migraines can themselves lead to significant psychological stress and subsequently can exacerbate the problem, (3) pain management strategies can assist migraineurs in coping with their pain more effectively, and (4) there exists a bidirectional association between mood disorders and migraines. The researchers also suggested that functional imaging studies could provide a physiological explanation for the processes by which psychological interventions in pain management function because studies have shown that changes in cognition and affect alter how the brain processes pain. For example, Bantick et al. [39], building on the copious support in the literature that pain perception is reduced when distraction techniques are utilized, used functional magnetic resonance imaging ( $\mathrm{fMRI}$ ) to examine the neural systems and mechanisms involved when participants were distracted while being subjected to painful stimuli. Bantick et al. [39] found that cognitively demanding tasks (an analog version of the Stroop test was used) increased the activation in the affective division of the anterior cingulate cortex (ACC) and 
orbitofrontal areas and reduced the activation in areas of the pain matrix such as the thalamus and insula. They also found that although the areas of the brain involved in sensory components of pain remained stable across time, activation in areas of the brain related to cognitive-emotional components of pain increased across time. Their findings supported the behavioral results of a reduction in pain perception, and they surmised that a patient's thoughts, emotions, and pain experiences alter how the brain interpreted pain. Phan et al. [40] conducted a meta-analysis of emotion activation studies using PET and $\mathrm{FMRI}$ and concluded that several regions of the brain associated with pain processing are involved with emotions and other psychological processes such as attention. Thus, as Nicholson et al. [31] and Brown et al. [34] reported, modulating pain by psychological factors could occur in these shared circuits and consequently alter the pain signal within the brain. Psychological interventions can also influence pain intensity. Garland [41] noted that the activation of a part of the brain implicated in emotion, the ventrolateral cortex, was positively associated with the extent to which pain was perceived to be controllable and negatively correlated with subjective pain intensity.

Garland [41] examined the notion that pain involved a process of cognitive appraisal where an individual evaluated the significance or meaning of sensory signals from her/his body and assessed whether or not there was harm. He stated that this appraisal was subjective and possibly determined by a neurobiological dissociation between sensory and affective aspects of the pain experience. He provided an example of changes in pain intensity, resulting in altered activation of the somatosensory cortex and changes in pain unpleasantness, resulting in altered activation of the ACC. Garland [41] surmised that whether an individual interpreted a sensation as threatening or not was dependent on whether the person believed that he/she could deal with the sensation or not. If available coping resources were sufficient to deal with the sensation during this complex cognitive process of appraisal, pain could be perceived as controllable. The result was that when the pain was cognitively perceived to be controllable, pain intensity was reduced, irrespective of whether the person acted to control the pain [41]. When the pain was interpreted as a harmless sensation, for example, warmth, there was an increased likelihood that the individual would feel as though he/she had control over the pain. As Garland explained, psychological interventions could reduce pain severity by altering the interpretation of pain sensations such that they were interpreted as innocuous sensory information. Conversely, he explained that when the pain was perceived as overwhelming and uncontrollable (pain catastrophizing), there was an association with increased pain intensity, irrespective of the physical trauma. Pain catastrophizing could therefore be considered an important factor in treating migraines.

\section{Personality}

Personality traits have also been involved in migraineurs. Hines [12] described the research conducted by Wolff [4] as a "thorough study of the personality features and reactions of patients who had migraine" (p. 989). He listed the features of these patients as "hardworking, hard drivers, and have the ability to accomplish much in a short space of time" (p. 989) and reported that the features led to resentment when things did not go according to the plan. He posited that this resentment caused emotional upset, which was subsequently likely to precipitate a migraine. Maizels et al. [7] suggested a bidirectional relationship between migraines and mood. They stated that the stress of chronic headaches could cause mood changes, for example, anxiety. A review of 
personality traits, personality disorders, and migraines [42] found that individuals who suffered from migraines frequently endorsed higher levels of neuroticism than individuals without migraines. This finding was consistent with that from a review by Silbersetin et al. [43]. No other personality traits were found to be associated as much with migraineurs.

\section{Pain Catastrophizing}

Haythornthwaite [44] described catastrophizing as the cognitive and emotional responses to pain which is negative in nature and typified by rumination, magnification of pain, and helplessness. She reported a strong correlation between catastrophizing and disability, independent of pain severity. Sullivan et al. [45] developed the Pain Catastrophizing Scale (PCS) and found that the PCS, as an instrument for measuring catastrophizing in pain patients, is a valuable, reliable, and valid tool. Furthermore, they noted the ability of the PCS to significantly predict the intensity of both emotional and physical distress of individuals participating in a cold pressor task and an electrodiagnostic evaluation. It was also noted that catastrophizing remained stable over time unless intervention was undertaken to address it.

Catastrophizing in patients with migraines has been associated with impaired functioning and quality of life, independent of migraine characteristics and other psychological variables [46]. However, Chiros and O'Brien [47] studied 74 individuals with migraine headaches and observed that pain-related acceptance reduced the impact of catastrophizing and led to an increase in daily activities.

Thorn et al. [48] cited several studies that illustrated that migraineurs, as well as individuals suffering from tension-type headaches, endorsed more catastrophic thoughts when coping with painful events than individuals who did not suffer from either condition. Thorn et al. [48] evaluated the efficacy of CBT in 40 participants who had been diagnosed with either tension-type headaches or migraines and, among other measures, monitored changes in pain catastrophizing. A total of 31 participants completed their RCT, and in this instance, treatment was not found to influence pain catastrophizing. The researchers suggested that this may be because the mean PCS score for their patients was lower than the average reported by O'Sullivan et al. for patients enrolled in a multidisciplinary treatment program. Thorn et al. [48] explained that none of their participants scored in the 80th percentile, which, according to Sullivan et al., was indicative of patients who showed poor progress in pain rehabilitation programs.

Keefe et al. [49] described pain catastrophizing as the "tendency to focus on pain and negatively evaluate one's ability to deal with pain" (p. 196). These researchers summarized the research on catastrophizing and found that patients who catastrophized had long hospitalizations, high rates of healthcare usage, high levels of psychological distress, and high levels of pain-related disability. They also quoted the theory of Sullivan et al. that pain catastrophizing was a coping response, which elicited support from others.

High scores on the PCS are linked with high levels of disability [49], but changes in catastrophizing are associated with improvements in disability. For example, Scott et al. [50] treated 166 occupationally disabled individuals who had subacute pain in a 7-week multidisciplinary rehabilitation program to promote functional recovery. They measured PCS scores before and after the intervention and found that PCS reductions of $38 \%$ to $44 \%$ postintervention were best associated with return to work and low pain intensity ratings. 
Given the cyclical relationship between stress and migraines and the notion that stress could be both a cause and effect of the social and medical disabilities caused by migraines [51], there is a strong argument for interventions such as hypnotherapy where the focus of treatment is to reduce stress by targeting components of pain such as unhelpful cognitions (e.g., catastrophizing) and behavior (e.g., activity avoidance). The neurological and clinical research literature strongly suggests that hypnosis can alter how emotion is experienced by patients and subsequently impacts sensation, behavior, and cognition [52]. Furthermore, hypnosis is safe and is devoid of the negative side effects that accompany many pharmacological treatments [53].

Considering these findings, and given the low adherence to OMPMs, possibly due to adverse effects or inefficacy [54], it may be prudent to take the suggestion of Edmeads [9] that we venture outside the vascular versus neurological debate and go one step further. By examining the influence of social and psychological factors in addition to biological factors, we can then better understand the etiology and pathophysiology of migraines and consequently offer improved treatment programs such as hypnosis, which is described by Kittle and Spiegel [53] in The American Journal of Medicine as "the most effective treatment you have yet to prescribe."

\section{Author Contributions}

The author did all the research work of this study.

\section{Competing Interests}

The authors have declared that no competing interests exist.

\section{References}

1. Hepp Z, Dodick DW, Varon SF, Chia J, Matthew N, Gillard P, et al. Persistence and switching patterns of oral migraine prophylactic medications among patients with chronic migraine: $\mathrm{A}$ retrospective claims analysis. Cephalalgia. 2017; 37: 470-485.

2. Benoit DP. An introduction to migraine pathophysiology. Tech Reg Anesth Pain Manag. 2009; $13:$ 5-8.

3. Willis T. Cerebri anatome: Cui accessit nervorum descriptio et usus. London: Typis Ja. Flesher, impensis Jo. Martyn \& Ja; 1664.

4. Wolff HG. Personality features and reactions of subjects with migraine. Archives Neurol Psychiatry. 1937; 37: 895-921.

5. Blau JN. Migraine pathogenesis: The neural hypothesis reexamined. J Neurol Neurosurg Psychiatry. 1984; 47: 437-442.

6. Welch KM. Migraine: A biobehavioral disorder. Arch Neurol. 1987; 44: 323-327.

7. Maizels M, Aurora S, Heinricher M. Beyond neurovascular: Migraine as a dysfunctional neurolimbic pain network. Headache. 2012; 52: 1553-1565.

8. Goltman AM. The mechanism of migraine. J Allergy. 1936; 7: 351-355.

9. Edmeads J. What is migraine? Controversy and stalemate in migraine pathophysiology. J Neurol. 1991; 238: S2-S5.

10. Goadsby PJ. Pathophysiology of headache. In: Wolff's Headache and Other Head Pain. 7th ed. New York, NY: Oxford University Press; 2001. pp.57-72. 
11. Goadsby PJ. Recent advances in the diagnosis and management of migraine. BMJ. 2006; 332: 25-29.

12. Hines EA. Migraine. Am J Nurs. 1938; 38: 988-992.

13. Weiller C, May A, Limmroth VA, Jüptner M, Kaube $H$, Schayck RV, et al. Brain stem activation in spontaneous human migraine attacks. Nat Med. 1995; 1: 658-660.

14. Liveing E. On megrim, sick-headache, and some allied disorders. A contribution to the pathology of nerve-storms. London: J \& A Churchill. 1873.

15. Hamel E, Currents $H$. Serotonin and migraine: Biology and clinical implications. Cephalalgia. 2007; 27: 1293-1300.

16. Yakhnitsa VA, Pilyavskii Al, Limansky YP, Bulgakova NV. Modulation of the activity of midbrain central gray substance neurons by calcium channel agonists and antagonists in vitro. Neuroscience. 1996; 70: 159-167.

17. D'Andrea G, D'Arrigo A, Dalle Carbonare M, Leon A. Pathogenesis of migraine: Role of neuromodulators. Headache. 2012; 52: 1155-1163.

18. Welch KM, Nagesh V, Aurora SK, Gelman N. Periaqueductal gray matter dysfunction in migraine: Cause or the burden of illness? Headache. 2001; 41: 629-637.

19. Raskin NH, Hosobuchi Y, Lamb S. Headache may arise from perturbation of brain. Headache. 1987; 27: 416-420.

20. Mathew NT. Pathophysiology of chronic migraine and mode of action of preventive medications. Headache. 2011; 51: 84-92.

21. Reisman M, Fuller CJ. Is patent foramen ovale closure indicated for migraine? Patent foramen ovale closure for migraine. Circ Cardiovasc Interv. 2009; 2: 468-474.

22. Pietrobon D, Striessnig J. Neurobiology of migraine. Nat Rev Neurosci. 2003; 4: 386-398.

23. Harder B. Against the migraine: A procedure's serendipitous success hints that some headaches start in the heart. Sci News. 2005; 167: 119-120.

24. Ducros A. Genetics of migraine. Rev Neurol. 2013; 169: 360-371.

25. Boldig K, Butala N. Migraines and CGRP monoclonal antibodies: A review of car-diovascular side effects and safety profile. Int J Neurol Neurother. 2020; 7: 101.

26. Boorsook D, Maleki N, Burstein R. Migraine. In: Neurobiology of Brain Disorders. Biological Basis of Neurological and Psychiatric Disorders. Cambridge, MA: Academic Press; 2015. pp.693-708.

27. Charles AC, Baca SM. Cortical spreading depression and migraine. Nat Rev Neurol. 2013; 9: 637644.

28. Close LN, Eftekhari S, Wang M, Charles AC, Russo AF. Cortical spreading depression as a site of origin for migraine: Role of CGRP. Cephalalgia. 2019; 39: 428-434.

29. Haanes KA, Edvinsson L, Sams A. Understanding side-effects of anti-CGRP and anti-CGRP receptor antibodies. J Headache Pain. 2020; 21: 26.

30. Cohen JM, Ning X, Kessler Y, Rasamoelisolo M, Campos VR, Seminerio MJ, et al. Immunogenicity of biologic therapies for migraine: A review of current evidence. J Headache Pain. 2021; 22: 3.

31. Nicholson RA, Houle TT, Rhudy JL, Norton PJ. Psychological risk factors in headache: CME. Headache. 2007; 47: 413-426.

32. Merikangas KR, Angst J, Isler H. Migraine and psychopathology: Results of the Zurich cohort study of young adults. Arch Gen Psychiatry. 1990; 47: 849-853.

33. Breslau N, Davis GC, Andreski P. Migraine, psychiatric disorders, and suicide attempts: An epidemiologic study of young adults. Psychiatry Res. 1991; 37: 11-23. 
34. Brown $\mathrm{H}$, Newman $\mathrm{C}$, Noad R, Weatherby S. Behavioural management of migraine. Ann Indian Acad Neurol. 2012; 15: S78-S82.

35. Hamelsky SW, Lipton RB. Psychiatric comorbidity of migraine. Headache. 2006; 46: 1327-1333.

36. Swanson SA, Zeng $Y$, Weeks $M$, Colman I. The contribution of stress to the comorbidity of migraine and major depression: Results from a prospective cohort study. BMJ Open. 2013; 3: e002057.

37. Henryk-Gutt R, Rees WL. Psychological aspects of migraine. J Psychosom Res. 1973; 17: 141-153.

38. Kim S, Bae DW, Park SG, Park JW. The impact of Pain-related emotions on migraine. Sci Rep. 2021; 11: 577.

39. Bantick SJ, Wise RG, Ploghaus A, Clare S, Smith SM, Tracey I. Imaging how attention modulates pain in humans using functional MRI. Brain. 2002; 125: 310-319.

40. Phan KL, Wager T, Taylor SF, Liberzon I. Functional neuroanatomy of emotion: A meta-analysis of emotion activation studies in PET and fMRI. Neuroimage. 2002; 16: 331-348.

41. Garland EL. Pain processing in the human nervous system: A selective review of nociceptive and biobehavioral pathways. Prim Care. 2012; 39: 561-571.

42. Davis RE, Smitherman TA, Baskin SM. Personality traits, personality disorders, and migraine: $A$ review. Neurol Sci. 2013; 34: S7-S10.

43. Silberstein S, Lipton R, Breslau N. Migraine: Association with personality characteristics and psychopathology. Cephalalgia. 1995. 15:358-369.

44. Haythornthwaite J. It's a belief. It's an appraisal. It's coping... No, it's catastrophizing. In Current Topics in Pain: 12th World Congress on Pain. Seattle: IASP Press; 2009. pp.271-287.

45. Sullivan MJ, Bishop SR, Pivik J. The pain catastrophizing scale: Development and validation. Psychol Assess. 1995; 7: 524-532.

46. Holroyd KA, Drew JB, Cottrell CK, Romanek KM, Heh V. Impaired functioning and quality of life in severe migraine: The role of catastrophizing and associated symptoms. Cephalalgia. 2007; 27: 1156-1165.

47. Chiros C, O'Brien WH. Acceptance, appraisals, and coping in relation to migraine headache: An evaluation of interrelationships using daily diary methods. J Behav Med. 2011; 34: 307-320.

48. Thorn BE, Pence LB, Ward LC, Kilgo G, Clements KL, Davis AM, et al. A randomized clinical trial of targeted cognitive behavioral treatment to reduce catastrophizing in chronic headache sufferers. J Pain. 2007; 8: 938-949.

49. Keefe FJ, Rumble ME, Scipio CD, Giordano LA, Perri LM. Psychological aspects of persistent pain: Current state of the science. J Pain. 2004; 5: 195-211.

50. Scott W, Wideman TH, Sullivan MJ. Clinically meaningful scores on pain catastrophizing before and after multidisciplinary rehabilitation: A prospective study of individuals with subacute pain after whiplash injury. Clin J Pain. 2014; 30: 183-190.

51. Malone CD, Bhowmick A, Wachholtz AB. Migraine: Treatments, comorbidities, and quality of life, in the USA. J Pain Res. 2015; 8: 537-547.

52. Nash MR, Barnie AJ. The Oxford handbook of hypnosis: Theory, research, and practice. Oxford: Oxford University Press; 2012.

53. Kittle J, Spiegel D. Hypnosis: The most effective treatment you have yet to prescribe. Am J Med. 2021; 134: 304-305.

54. Hepp Z, Bloudek LM, Varon SF. Systematic review of migraine prophylaxis adherence and persistence. J Manag Care Pharm. 2014; 20: 22-33. 


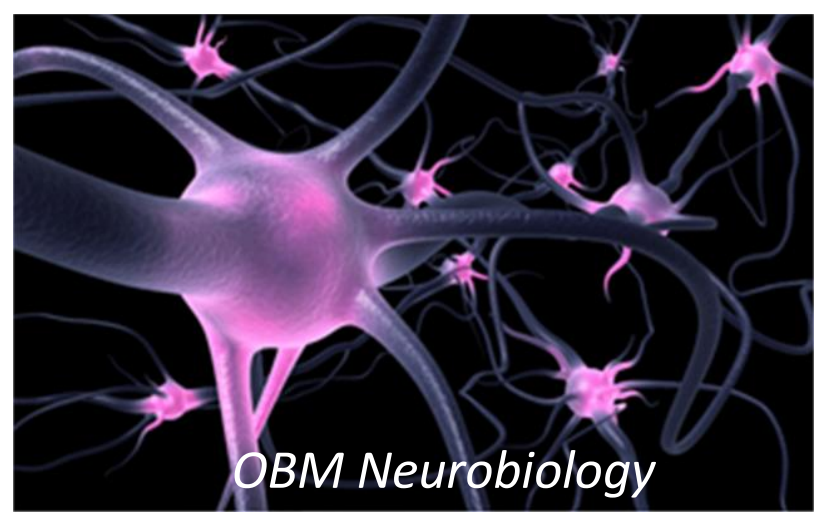

Enjoy OBM Neurobiology by:

1. Submitting a manuscript

2. Joining volunteer reviewer bank

3. Joining Editorial Board

4. Guest editing a special issue

For more details, please visit:

http://www.lidsen.com/journals/neurobiology 